\title{
Competencias que pueden Desarrollarse en la Asignatura de Mecánica en el Currículo de Ingeniería
}

\author{
María D. Rodríguez, Daniel A. Mena, Carlos M. Rubio \\ Facultad de Ingeniería Química, Universidad Autónoma de Yucatán, Periférico Nte. Km. 33.5, \\ Tablaje Catastral, 13615, Col. Chuburná de Hidalgo inn, C.P. 97203, Mérida, Yucatán-México \\ (e-mail: mmartin@uady.mx)
}

Recibido Feb. 18, 2011; Aceptado Mar. 29, 2011; Versión final recibida May. 02, 2011

\begin{abstract}
Resumen
En este trabajo se adaptó el programa de la asignatura Mecánica Clásica al enfoque de competencias haciendo el análisis y selección de las competencias genéricas a desarrollar. La experiencia se enmarca en el compromiso de la Facultad de Ingeniería Química de la Universidad Autónoma de Yucatán en México de formar ingenieros de calidad, capaces de desempeñarse exitosamente tanto en el campo industrial como en la academia. Se incluyeron los conocimientos y las habilidades a adquirir ordenados en una secuencia que se consideró idónea para desarrollar la competencia correspondiente. Se recomienda que para implantar un modelo como el descrito se den cursos de capacitación e inducción, tanto para profesores como para alumnos. Esto porque la adopción del modelo implica un cambio conceptual importante con respecto al modelo tradicional y que no es fácilmente comprendido.
\end{abstract}

Palabras clave: ciencias básicas, competencia, mecánica clásica, parámetros, indicadores

\section{Skills that can be Developed in the Course of Classical Mechanics in the Engineering Curriculum}

\begin{abstract}
The program of the course Classical Mechanics has been adapted to the competence approach by analyzing and selecting the generic skills to be developed during the course. The experiment is part of the commitment of the School of Chemical Engineering of the Autonomous University of Yucatan in Mexico to form qualified engineers, able to successfully perform in both the industry and the academia. Knowledge and skills were included in a sequence that was considered to be suitable for developing the corresponding competence. It is recommended that when implementing a model such as the one described in this paper, induction and training courses, for both students and instructors, should be delivered. This because the implementation of the model represents an important conceptual change with respect to the traditional model, and it is not easily comprehended
\end{abstract}

Keywords: basic sciences, competence, classical mechanics, parameters, indicators 


\section{INTRODUCCIÓN}

El desarrollo de la sociedad industrializada caracterizada por la masiva producción de bienes manufacturados con el uso intensivo de máquinas en reemplazo de la fuerza humana, ha progresado paulatinamente hacia una sociedad de información y conocimiento, caracterizada por el predominio de la industria de los servicios. En ésta última se pone de manifiesto la necesidad de contar con personas que, además de poseer habilidad manual, tengan otro tipo de capacidades que les permita tomar parte activa de las decisiones en el lugar de trabajo en que se desempeñan (Vargas, 2000). Esto ha tenido una serie de repercusiones en todos los ámbitos, incluyendo el educativo. La educación en ingeniería en los últimos años ha evidenciado la necesidad de combinar las habilidades duras tradicionales con las habilidades blandas que promuevan la integridad del profesional (Shuman et al, 2005).En los Estados Unidos de Norteamérica, por más de 70 años las acreditaciones han garantizado el control de calidad en la educación de las ingenierías, buscando asegurar que los graduados de programas acreditados están preparados para la práctica profesional. Sin embargo, en la década de los 80 los criterios utilizados eran muy rigurosos y prescriptivos, inhibiendo el desarrollo de programas innovadores que reflejaran los cambios de las necesidades de la práctica. No es hasta el año 1995 que el ABET (Accreditation Board for Engineering and Technology) y las partes interesadas desarrollaron nuevos criterios (EC2000), los cuales privilegian los resultados del aprendizaje, la evaluación y la mejora continua sobre las especificaciones detallas de los currículos. Estos criterios, junto con los acuerdos internacionales facilitarán el incremento de la movilidad profesional global.

El EC2000 ha impactado los sistemas de acreditación de muchos países (Prados et al, 2005). Los nuevos criterios (EC2000) se estipula que para acreditar un currículo de ingeniería el programa debe demostrar que los graduandos tienen una serie de habilidades y conocimientos (p.ej. diseñar, desarrollar, implementar, y mejorar sistemas integrados que incluyen personas, materiales, información, equipos y energía) necesarios para demostrar su competencia laboral en dicha profesión. Si bien el criterio mencionado por la ABET es un requisito y no una declaración de competencias de egreso, queda clara la orientación formativa que se le debe inculcar al estudiante de ingeniería. Para tal efecto, esta misma agencia de acreditación de Estados Unidos establece requisitos que los programas curriculares deben cumplir en relación a las habilidades que deben asegurar en los graduados, como por ejemplo: la habilidad para aplicar conocimientos de matemáticas, ingeniería y ciencias; habilidad para diseñar y conducir experimentos así como para analizar e interpretar datos; entendimiento de las responsabilidades profesionales y éticas; habilidad para comunicarse efectivamente (Elizandro y Matson, 2001). Estos requisitos evidencian una relación directa con las competencias genéricas propuestas por Tuning (Gonzales y Wagenaar, 2003).

Para Pinilla (2005), La misión del proceso educativo es formar personas con valores para poder convivir en sociedad, "saber- ser", que además tengan conocimientos en áreas específicas del conocimiento, "saber", pero que también sepan hacer tareas o labores para sí mismos y para los demás, "saber hacer", así como ser creativos,"saber emprender".En la literatura existen múltiples definiciones al concepto de competencia, sin embargo en algunos estudios comparativos, como el de Méndez y Roegiers (2005), se identifica que diversos autores coinciden en que el concepto encierra componentes fundamentales que se traducen como recursos (conocimientos, aptitudes y comportamientos) que se movilizan para resolver una tarea compleja que exige una situación problema de una disciplina y que debe ser evaluable. El informe final del proyecto Tuning América Latina (2007) menciona en una de sus conclusiones que existe un acuerdo general entre los participantes de la importancia del concepto de competencia a la hora de elaborar o perfeccionar un currículo.

Una competencia puede definirse en términos de cinco características esenciales (Roegiers, 2007):

1. Movilización de un conjunto de recursos: la competencia requiere la movilización de un conjunto de recursos (conocimientos, saberes de experiencia, esquemas, automatismos, capacidades, saberes-hacer de diferentes tipos, saberes-ser, etc.). Generalmente estos recursos forman un 
conjunto tan integrado que es difícil analizar todos los recursos movilizados durante el ejercicio de una competencia.

2. Carácter finalizado: la competencia tiene una función social y una utilidad social desde el punto de vista del individuo que la posee.

3. Familia de situaciones: la movilización de recursos se hace en relación con una familia bien determinada de situaciones.

4. Carácter disciplinario: la competencia es definida a través de una categoría de situaciones, correspondiente a problemas específicos ligados a una disciplina y directamente de las exigencias de ésta.

5. Evaluación: la competencia es evaluable, ya que puede medirse la calidad de la ejecución de la tarea y la calidad del resultado, aunque se limita a una familia de situaciones.

Una clasificación empleada en el ámbito universitario, de acuerdo al proyecto Tuning para América Latina (Delgado García ET AL., 2005) se muestra en la Tabla 1.

Tabla 1: Definición de los distintos tipos de competencias empleadas en el ámbito universitario

\begin{tabular}{|c|c|c|}
\hline \multicolumn{2}{|c|}{ Tipos de Competencias } & Definiciones \\
\hline 1. & $\begin{array}{l}\text { Competencias } \\
\text { transversales: }\end{array}$ & $\begin{array}{l}\text { Son aquellas competencias compartidas por todas las materias o ámbitos } \\
\text { de conocimiento; también son conocidas como competencias genéricas. }\end{array}$ \\
\hline & 1.1. Instrumentales: & $\begin{array}{l}\text { Son aquellas que miden las capacidades y la formación del egresado; } \\
\text { constituyen un medio para obtener un determinado fin. Ejemplos de este } \\
\text { tipo de competencias son las capacidades de análisis y síntesis, de } \\
\text { organización y planificación, de comunicación oral y escrita en la propia } \\
\text { lengua. Incluyen también las habilidades básicas de manejo de la } \\
\text { computadora, de gestión de la información (búsqueda y análisis de } \\
\text { información proveniente de fuentes diversas), de resolución de problemas y } \\
\text { de toma de decisiones. }\end{array}$ \\
\hline & \multirow[t]{2}{*}{ 1.2. Personales: } & $\begin{array}{l}\text { Miden las habilidades de relación social y de integración en diferentes } \\
\text { colectivos y la capacidad de trabajar en equipos específicos y } \\
\text { multidisciplinares; se trata de las capacidades que permiten que las } \\
\text { personas tengan interacción con los demás. }\end{array}$ \\
\hline & & $\begin{array}{l}\text { Se refieren a la capacidad de crítica y autocrítica, de comunicación con } \\
\text { expertos de otras áreas, de trabajo en equipo, de apreciación de la } \\
\text { diversidad y de la multiculturalidad, a la habilidad para trabajar en un } \\
\text { contexto internacional y el compromiso ético. }\end{array}$ \\
\hline & 1.3. Sistémicas: & $\begin{array}{l}\text { Son las destrezas asociadas con la comprensión de la totalidad de un } \\
\text { sistema; se asocian con las cualidades individuales tales como las } \\
\text { capacidades para aplicar los conocimientos en la práctica, para aprender, } \\
\text { para adaptarse a nuevas situaciones, para generar nuevas ideas } \\
\text { (creatividad), de diseño y gestión de proyectos, de liderazgo; a la habilidad } \\
\text { de investigar, de trabajo autónomo, a la motivación hacia el logro y su } \\
\text { preocupación por la calidad. }\end{array}$ \\
\hline 2 & $\begin{array}{l}\text { Competencias } \\
\text { específicas }\end{array}$ & $\begin{array}{l}\text { Son aquellas relacionadas con disciplinas concretas y están más } \\
\text { relacionadas con los conocimientos. }\end{array}$ \\
\hline & 2.1. Académicas: & Se refieren a los conocimientos teóricos de la disciplina (conocer) \\
\hline & 2.2. Disciplinares: & $\begin{array}{l}\text { Es el conjunto de conocimientos prácticos requeridos en cada sector } \\
\text { profesional (hacer). }\end{array}$ \\
\hline & 2.3. Profesionales: & $\begin{array}{l}\text { Incluyen habilidades de comunicación e investigación aplicadas al ejercicio } \\
\text { de una profesión (saber-hacer). }\end{array}$ \\
\hline
\end{tabular}


De acuerdo a un estudio realizado en los Estados Unidos de Norteamérica (Martin et al, 2005), se confirmó que las habilidades técnicas son la base de la ingeniería pero las de comunicación, el trabajo en equipo y las relaciones interpersonales también son importantes en el lugar de trabajo. Las habilidades no técnicas deben de ser tomadas en cuenta en el desarrollo del currículo y no deben de ser pensadas en forma aislada. Davis y colaboradores (2005) desarrollaron un proceso y definieron un perfil para las carreras de ingeniería destacando en el perfil las habilidades técnicas, interpersonales y las profesionales. El primer año de los estudiantes en una universidad es crítico, ya que de su adaptación y motivación dependerá su éxito académico. Facione (1997) en su trabajo Critical thinking assessment in nursing education programs: An agregate data analysis menciona que el desarrollo de la mayoría de las habilidades de pensamiento ocurre en los primeros 2 años (Reason et al, 2006). La Universidad Autónoma de Yucatán (UADY) es una institución pública de educación superior de gran prestigio académico. Es miembro del Consorcio de Universidades Mexicanas (CUMEX), el cual agrupa a las Universidades con una alta competitividad académica en México. La Facultad de Ingeniería Química es una dependencia de la UADY con tres programas de licenciatura acreditados en el área de la ingeniería y la química con una matrícula de 695 alumnos en ellos.

\section{METODOLOGÍA}

La misión de las Ciencias Básicas en Ingeniería es coadyuvar a la formación integral de los alumnos, proporcionando conocimientos de matemáticas, física y química necesarios para continuar en forma exitosa sus estudios de licenciatura; y, de manera transversal, colaborar en inculcarles habilidades de razonamiento, valores éticos, conciencia social y ecológica, espíritu crítico, liderazgo y deseos de aprender de manera permanente por sí mismos; fomentar el trabajo en equipo, la adquisición de aptitudes, actitudes y valores para ser agentes promotores de cambio y beneficio social. Conforme al planteamiento de las competencias genéricas que se espera desarrollen los alumnos de ingeniería en las asignaturas básicas, se promueve el fomento a la capacidad de análisis y síntesis que se manifiesta en el saber plantear y resolver problemas utilizando los conocimientos obtenidos en el programa de estudios. Al desarrollar la capacidad anterior se promueven habilidades cognoscitivas y capacidades metodológicas que permiten a los estudiantes tener un pensamiento capaz de comprender y manipular ideas para la correcta toma de decisiones en los problemas de aplicación que se plantean a este nivel y que representan un ensayo de la problemática a la que se enfrentarán en la vida laboral.

Con base en las definiciones de la tabla 1, para identificar las competencias transversales en sus tres tipos (instrumentales, personales y sistémicas) y sus posibles obstáculos, se revisaron los objetivos, los contenidos y los mecanismos de evaluación del programa de estudio de la asignatura seleccionada. Para el caso de las instrumentales, se verificó que el programa contuviera explícitamente el uso de software y/o la elaboración de trabajos escritos tales como proyectos, ensayos, etc. El trabajo en equipo es un indicador del desarrollo de las competencias personales $\mathrm{y}$, finalmente, la elaboración de proyectos $\mathrm{y} / \mathrm{o}$ propuestas de proyectos de investigación identificarán la promoción de competencias sistémicas. Adicionalmente, al considerar las características básicas propuestas por Roegiers (2007) para las competencias, en la revisión de los objetivos generales y específicos de los programas de estudio se verificará que los niveles taxonómicos de los mismos sean al menos de aplicación, puesto que una competencia implica la movilización de recursos, el carácter finalizado de las mismas y la posibilidad de ser evaluadas, además de su carácter disciplinario.

La asignatura tratada en este trabajo como referente, impartida en el tronco común de todas las ingenierías, es Mecánica Clásica (Física II). Los autores la eligieron para hacer una primera adaptación del programa de una asignatura de ciencias básicas al enfoque de competencias debido a la característica integradora de la Física en este nivel, a saber, además de que los conceptos físicos, requiere el uso intensivo de las Matemáticas. Las herramientas matemáticas se utilizan y refuerzan en el manejo de las leyes que rigen el movimiento. Se analizó el programa de la asignatura y las competencias específicas y transversales incluidas. A continuación se muestra el programa explícito de las cinco unidades en las que se divide la asignatura Física II. 
Física II. Objetivos generales: En el nivel temático, el alumno comprenderá los fundamentos de la mecánica clásica, los sistemas de fuerzas, el movimiento, trabajo y energía. En el nivel formativo, el alumno debe desarrollar capacidades y habilidades para resolver problemas, abstractos y de aplicación utilizando los diferentes sistemas de unidades, principios y leyes relacionados con la estática, la cinemática, así como la dinámica lineal y circular (inercia, impulso, trabajo y energía) de partículas y cuerpos.

Se aplican cuatro exámenes, uno por cada unidad del programa y cada uno con un valor de 10 puntos. Las tareas son ejercicios y proyectos de diseño (cálculos y diseño básico de modelos en donde se visualice la acción del fenómeno físico a estudiar) selectos para cada unidad. Las tareas se califican en una escala numérica tomando en cuenta la calidad y el cumplimiento en la entrega de las mismas. La ponderación de estas actividades es: Exámenes 40 \%, Tareas 45 \%, y Proyecto final $15 \%$. El programa de la asignatura se detalla como sigue:

Unidad 1. Cinemática de la Partícula

Objetivo: Al finalizar la unidad el alumno podrá resolver problemas relacionados con la cinemática de partículas tanto en movimiento rectilíneo como en movimiento curvilíneo

1.1 Movimiento unidimensional

1.2.- Movimiento bidimensional tridimensional

Unidad 2. Cinética de la Partícula

Objetivo: Al finalizar la unidad el alumno podrá resolver problemas relacionados con la cinética de partículas tanto en movimiento rectilíneo como en movimiento curvilíneo

2.1. Fuerza y Leyes de Newton

2.2. Dinámica de la partícula

Unidad 3. Cinemática de partículas: Métodos de la energía de la cantidad de movimiento

Objetivos: Al finalizar la unidad, el alumno: i) Podrá resolver problemas de cinética de partículas aplicando el Principio del Trabajo y la Energía o la Ley de Conservación de la Energía según requiera el caso; y ii) Podrá resolver problemas de cinética de partículas en los que intervengan el Impulso y la cantidad de Movimiento

3.1 Trabajo y energía

3.2. Conservación de la energía

3.3. Colisiones

Unidad 4. Cinemática de cuerpos rígidos

Objetivos: Al finalizar la unidad, el alumno podrá: i) Resolver problemas que impliquen la traslación de un cuerpo rígido; ii) Resolver problemas de rotación de un cuerpo rígido; y iii) Resolver problemas que involucren el movimiento plano general de un cuerpo rígido

4.1. Traslación

4.2. Rotación

4.3. Movimiento plano general

Unidad 5. Movimiento plano de cuerpos rígidos: Fuerzas y Aceleraciones

Objetivo: Al finalizar la unidad el alumno podrá aplicar las leyes de la cinemática y la dinámica rotacional para explicar y predecir el movimiento de cuerpos rígidos

5.1. Movimiento plano de cuerpos rígidos

5.2. Métodos de la Energía y la Cantidad de Movimiento

Se procedió a la selección de las competencias genéricas y de las específicas y se eligió trabajar con una de la unidad 5. Dicha unidad se seleccionó, para ser analizada, por requerir todos los conocimientos y habilidades adquiridos en las 4 unidades anteriores. A saber: para entender el concepto de ecuaciones de movimiento para cuerpos rígidos es necesario haber dominado antes dicho concepto sobre partículas, recordando que en mecánica clásica una partícula representa un cuerpo de cualquier masa, concentrada ésta en un punto en el espacio, por lo que inicialmente no se hacen consideraciones (unidades 1,2 y 3 ) sobre el volumen y la forma del cuerpo que se mueve. Una vez construidos los conocimientos relativos a cinemática (descripción del movimiento 
sin tomar en cuenta las fuerzas que lo causan), cinética (aplicación de la segunda ley de Newton y sus consecuencias para predecir fuerzas y movimientos) y métodos energéticos para partículas (relaciones entre trabajo, energía y potencia junto con las leyes clásicas de conservación) declarados en las primeras 3 unidades se procede a modelar todo cuerpo rígido como un conjunto de partículas cuya masa se considera concentrada en su centro de masa, pero cuyo volumen debe ser ya considerado debido a la posibilidad de presentar movimientos de giro o rotación (torca). A partir de este modelo, todos los conceptos y ecuaciones relacionadas con el movimiento de partículas se generalizan a los cuerpos rígidos tanto en su cinemática tratada en la unidad 4, como en su cinética y métodos energéticos tratados en la unidad 5. Para alcanzar este manejo de conceptos físicos y poder predecir el movimiento al nivel en que se trata esta unidad, se transita por un gradual aumento a través de las 4 en el nivel y variedad de las herramientas matemáticas empleadas en la deducción, comprensión y uso de las ecuaciones. Se utilizan inicialmente el álgebra y el cálculo diferencial e integral, para luego requerir el uso del cálculo vectorial en los temas relacionados con movimiento en el plano y sólidos rígidos. Se resaltan a continuación las siguientes competencias:

Competencia específica determinante de la asignatura: Predecir el comportamiento de un cuerpo aplicando el concepto de fuerza y las leyes del movimiento. Puesto que el programa de la asignatura no está construido por competencias, esta competencia se extractó de los objetivos generales declarados para la asignatura y contiene todo lo enumerado en esos objetivos.

Competencia específica de la unidad 5: Aplicar las leyes de la cinemática y la dinámica rotacional para explicar y predecir el movimiento de cuerpos rígidos. Este es el objetivo declarado en el programa para esta unidad.

Durante el análisis se trató de explicitar los procesos metacognitivos involucrados en la determinación y secuencia de las habilidades requeridas y en la selección de los indicadores de proceso y resultados, incluyendo su descripción y sus parámetros. Se entiende por indicador de proceso "todo dato empírico- cualitativo o cuantitativo- recogido de forma sistemática en relación con unas metas o procesos que nos permiten estimar la productividad y/o funcionalidad de un sistema". Están relacionados con las preguntas acerca de la ejecución de las actividades, e indican cómo se realizan las actividades desde un punto de vista cualitativo y cuantitativo. Por otro lado, los indicadores de resultado: permiten conocer el logro de los objetivos del proyecto educativo (López, 2004). La competencia específica de la unidad analizada puede ser dividida en subcompetencias entendiendo que éstas son competencias más generales que relacionadas e integradas correctamente forman una competencia más específica y especializada. Para cada subcompetencia se definieron los parámetros en los cuáles se puede basar la evaluación de la adquisición de ésta. Gómez (2002) propone tres metodologías para realizar trabajo por competencias, y que son:

1.- Trabajo por proyectos: A partir de una situación problema se desarrollan procesos de aprendizaje y construcción de conocimiento, vinculados al mundo exterior y al contexto. Realza la importancia del aprendizaje autónomo. Los proyectos son de carácter interdisciplinario, con el propósito de que los alumnos busquen y relacionen conocimientos no discutidos en clase.

2.- Resolución de problemas: Permite activar, promover y valorar los procesos cognitivos cuando los problemas y tareas se diseñan creativamente.

3.- Fomento de habilidades de colaboración: Son el trabajo de equipo y aprendizaje colaborativo. Con evaluaciones continuas, comunicación efectiva entre los integrantes y esquemas de coevaluación se fomentan habilidades sociales como la solución de conflictos, colaboración, responsabilidad y cultura de trabajo.

Estas 3 metodologías son las que se usaron para construir los indicadores de resultados propuestos para las competencias transversales a desarrollar. Se requirió un análisis detallado de las competencias seleccionadas, usando como referencias: la experiencia docente de los autores, 
las necesidades de los educandos, el contexto educativo en que se aborda la asignatura y el marco teórico arriba mencionado.

\section{RESULTADOS Y DISCUSIÓN}

La Tabla 2 presenta algunos detalles y resultados de las competencias transversales/genéricas a desarrollar en la asignatura Física II.

Tabla 2: Competencias Transversales/Genéricas a desarrollar en la asignatura Física II

\begin{tabular}{|c|c|c|}
\hline $\begin{array}{l}\text { Competencias } \\
\text { Instrumentales }\end{array}$ & Indicadores de Proceso & Indicadores de Resultados \\
\hline $\begin{array}{l}\text { Capacidad de } \\
\text { análisis y síntesis }\end{array}$ & $\begin{array}{l}\text { 1) Discrimina información } \\
\text { 2) Construye gráficas } \\
\text { 3) Desarrolla aplicaciones }\end{array}$ & $\begin{array}{l}\text { 1) Entrega ensayos ordenados y claros } \\
\text { 2) Elabora gráficas de movimiento } \\
\text { 3) Resuelve problemas de movimiento y } \\
\text { energía e interpreta resultados }\end{array}$ \\
\hline $\begin{array}{l}\text { Habilidades de } \\
\text { gestión de } \\
\text { información ( } \\
\text { buscar y analizar } \\
\text { información de } \\
\text { fuentes diversas) }\end{array}$ & $\begin{array}{l}\text { 1) Selecciona y organiza información } \\
\text { de distintas fuentes } \\
\text { 2) Interpreta los conceptos y sus } \\
\text { aplicaciones } \\
\text { 3) Desarrolla diseños simples }\end{array}$ & $\begin{array}{l}\text { 1) Presenta trabajos con información } \\
\text { pertinente y estructurada, de varias } \\
\text { fuentes. } \\
\text { 2) Interpreta adecuadamente conceptos y } \\
\text { sus aplicaciones } \\
\text { 3) Construye aplicaciones de los conceptos }\end{array}$ \\
\hline Interpersonales & Proceso & Indicadc \\
\hline Trabajo en equipo & $\begin{array}{l}\text { 1) Conoce reglas y rol de actividades } \\
\text { 2) Respeta y apoya a sus } \\
\text { compañeros } \\
\text { 3) Entrega trabajos originales y } \\
\text { consensuados }\end{array}$ & $\begin{array}{l}\text { 1) Participa en actividades programadas } \\
\text { asumiendo el rol adecuado } \\
\text { 2) Desarrolla trabajo colaborativo } \\
\text { 3) Explica y fundamenta cualquier parte del } \\
\text { tema a tratar }\end{array}$ \\
\hline ético & $\begin{array}{l}\text { 1) Es puntual en el cumplimiento de } \\
\text { acuerdos del inicio de semestre } \\
\text { 2) Entrega trabajos de propia autoría } \\
\text { 3) Escucha con atención a sus } \\
\text { compañeros, respeta diferentes ideas } \\
\text { y creencias aun contrarias a las } \\
\text { propias }\end{array}$ & $\begin{array}{l}\text { 1) Responsabilidad, diálogo fluido en el } \\
\text { aula, proceso de enseñanza-aprendizaje } \\
\text { dinámico y ético } \\
\text { 2) Estudiantes y docentes son escuchados } \\
\text { 3) Respeto a las creencias } \\
\text { 4) Pulcritud en aulas e instalaciones, } \\
\text { pulcritud verbal y trabajos limpios }\end{array}$ \\
\hline Sistémicas & Indicadores de Proceso & Indicadores de Resultados \\
\hline $\begin{array}{l}\text { Capacidad } \\
\text { aprender }\end{array}$ & $\begin{array}{l}\text { 1) Posee motivación intrínseca y } \\
\text { conoce conceptos previos y nuevos } \\
\text { 2) Concibe aplicaciones del tema }\end{array}$ & $\begin{array}{l}\text { 1) Participa activa y consistentemente } \\
\text { 2) Desarrolla adecuadamente las tareas } \\
\text { 3) Diseña aplicaciones del tema }\end{array}$ \\
\hline
\end{tabular}

Para la Competencia específica que se ha analizado y desarrollado en este trabajo, se presentan de manera detallada cada una de las subcompetencias que la componen, con sus respectivos parámetros indicadores del proceso y los conocimientos, actitudes vinculados a cada una. Las actitudes que se deben desarrollar, son transversales a todos los conocimientos y habilidades que se adquieren en la unidad.

Competencia específica analizada y desarrollada: Aplicar las leyes de la cinemática y dinámica rotacional para explicar y predecir el movimiento. Se ha dividido en 5 subcompetencias que se presentan en la tabla 3, cada una con su descripción y su parámetro de evaluación. 
Tabla 3: Subcompetencias en las que se divide la competencia específica analizada

\begin{tabular}{|c|c|c|}
\hline $\begin{array}{l}\text { Sub- } \\
\text { competencia }\end{array}$ & Descripción & Parámetro de evaluación \\
\hline 1 & $\begin{array}{l}\text { Usa las matemáticas para modelar el } \\
\text { movimiento de acuerdo a las leyes de la } \\
\text { Física. Es transversal a todos los } \\
\text { conocimientos de la unidad. }\end{array}$ & Traslada el problema al lenguaje matemático \\
\hline 2 & $\begin{array}{l}\text { Determina las especificaciones del } \\
\text { movimiento }\end{array}$ & $\begin{array}{l}\text { Obtiene las condiciones iniciales y de frontera } \\
\text { del movimiento }\end{array}$ \\
\hline 3 & $\begin{array}{l}\text { Determina los ejes de movimiento de un } \\
\text { cuerpo }\end{array}$ & $\begin{array}{l}\text { Localiza los ejes de movimiento de un sólido } \\
\text { rígido respecto a distintos marcos de referencia }\end{array}$ \\
\hline 4 & $\begin{array}{l}\text { Determina las principales direcciones de } \\
\text { inercia del sólido rígido }\end{array}$ & $\begin{array}{l}\text { a)Localiza los ejes de movimiento de un sólido } \\
\text { rígido respecto a distintos marcos de referencia; } \\
\text { b) Encuentra el momento cinético }\end{array}$ \\
\hline 5 & $\begin{array}{l}\text { Predice las condiciones del movimiento } \\
\text { de rotación y traslación combinados de } \\
\text { un sólido rígido }\end{array}$ & $\begin{array}{l}\text { a) Traslada el problema al lenguaje matemático; } \\
\text { b) Obtiene las condiciones iniciales y de } \\
\text { frontera del movimiento; c) Localiza ejes de } \\
\text { movimiento respecto a marcos de referencia; c) } \\
\text { Encuentra el momento cinético; d) Compara la } \\
\text { predicción de los modelos con el movimiento } \\
\text { real de un sólido rígido }\end{array}$ \\
\hline
\end{tabular}

En la tabla 4 se detallan los conocimientos y actitudes asociados a la competencia específica de la unidad 5, así como su vinculación con cada una de las subcompetencias declaradas en la tabla 3. Se observa que al dividir la competencia específica en subcompetencias, el estudiante debe ir avanzando en el desarrollo de la competencia total, hasta lograr la integración completa de la competencia específica.

Tabla 4: Conocimientos y actitudes que forman parte de la competencia específica de la Unidad 5 y la subcompetencia vinculada a cada una de ellas

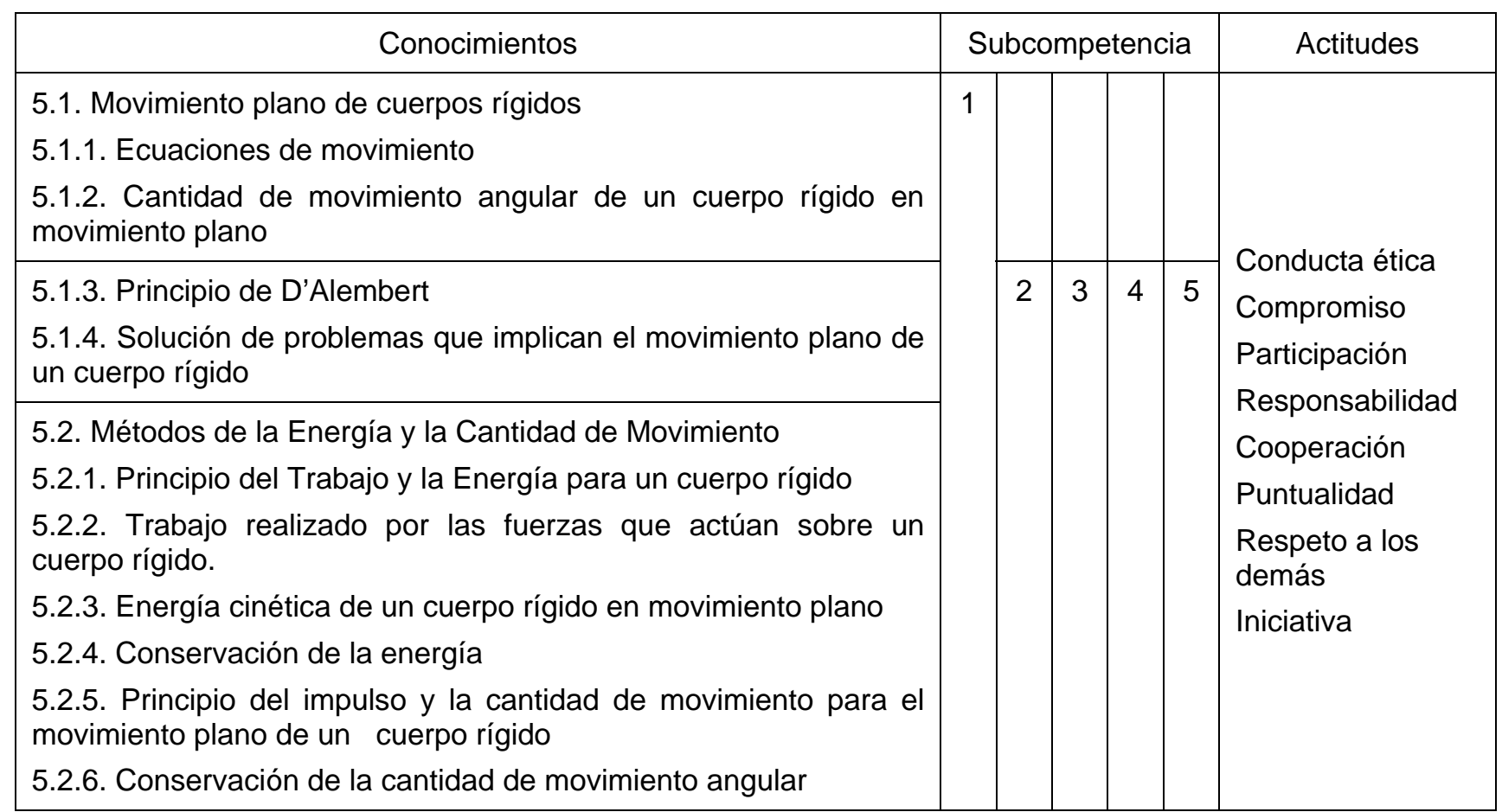


No obstante en el medio académico vinculado con las ciencias exactas y la ingeniería se habla de la dificultad de adaptar los programas de ciencias básicas al esquema de aprendizaje por competencias, en particular en lo referente a las competencias transversales (García et al , 2010) en este trabajo se muestra que haciendo un análisis detallado del concepto de competencia en educación y conociendo bien el perfil deseable a alcanzar tomando en cuenta la formación integral del estudiante, es posible reconocer las competencias implícitas en las asignaturas de ingeniería. El hecho de explicitar las competencias lleva a definir de manera sistemática las metodologías y estrategias a utilizar para desarrollar estas competencias, aterrizando finalmente en el diseño de un curso mucho más activo que los cursos tradicionales.

Es importante destacar que en la Facultad de Ingeniería química de la UADY aunque todavía no se adopte este modelo, desde hace mucho tiempo se ha fomentado el desarrollo de competencias en sus alumnos de forma implícita. Así, es posible replantear la definición de objetivos en los procesos de revisión de planes y programas. En la aplicación de este modelo se recomienda dar cursos de inducción y capacitación a los profesores y a los alumnos, debido a que es un cambio de paradigma con el modelo tradicional.

\section{CONCLUSIONES}

Del estudio y análisis presentado y los resultados obtenidos, se puede extrae las siguientes conclusiones principales:

i) La implementación de la formación por competencias demanda una transformación radical, de todo un paradigma educativo, implica cambios en la docencia, en la organización del sistema educativo, en la reflexión pedagógica y sobre todo en esquemas de formación arraigados por la tradición.

ii) Es necesario replantear la metodología didáctica usada en el aula para incorporar, por ejemplo, aprendizaje basado en problemas y aprendizaje basado en proyectos.

iii) Las competencias se deben desarrollar desde la perspectiva de un proceso de enseñanza aprendizaje que centre sus esfuerzos en el estudiante, por lo cual el profesor requiere de capacitación en metodologías y tecnologías educativas.

iv) Si se realiza un análisis detallado del concepto de competencia en educación y teniendo claro el perfil deseable del futuro profesional, es posible reconocer las competencias que pueden desarrollarse en las asignaturas de ingeniería.

\section{REFERENCIAS}

Bravo, N. Competencias Proyecto Tuning-Europa, Tuning-América Latina. (2009). http://acreditacion.unillanos.edu.co/contenidos/cpacitacion_docente_2semestre_2007/competenci as_proyectotuning.pdf, Acceso: 8 de marzo (2010)

Delgado, A., Borge, R., y otros. Competencias y diseño de la evaluación continua y final en el Espacio Europeo de Educación Superior. Catalunya: Ministerio de Educación y Ciencia. Dirección General de Universidades (2005).

Davis, D., Beyerlein, S. y Davis, I. Development and use of an engineer profile. Proceedings of the 2005 American Society for Engineering Education Annual Conference \& exposition. Session 3155. Oregon, junio 12 al 15 (2005).

Elizandro, D., Matson, J., Industrial Engineering Program Management in the ABET 2000 Enviroment. Proceedings of the 2001 ASEE/SEFI/TUB colloquium, ASEE. Berlin, septiembre 15 al 18 (2001). 
García, J., Vargas, G. y otros. Obstáculos para el desarrollo de competencias transversales en planes de estudio tradicionales. Ponencia presentada en el XXX Congreso de la Asociación Nacional de Facultades y Escuelas de Ingeniería. Chetumal, México (2010)

Gómez, J. Lineamientos pedagógicos para una educación por competencias. Capítulo: El concepto de competencia II. Una mirada interdisciplinar. Santa Fe de Bogotá: Sociedad Colombiana de Pedagogía (2002).

Gonzales, J. y Wagenaar, R., Tuning Educational Structures in Europe. Informe Final Fase Uno. Universidad de Deusto, España (2003).

López, A. Autoevaluación del Programa Educativo de Informática a través de indicadores (2004).http://www.ilustrados.com/publicaciones/EplplyppApjsmwYQtr.php Acceso: 30 de marzo 2011

Martin, R., Maytham, B., Case, J. y Fraser, D. engineering graduates' perceptions of how well they were prepared for work in industry. European Journal of Engineering Education, 30(2), 167-180 (2005).

Méndez, A. y Roegiers, X., Terminología pedagógica específica al enfoque por competencias: El concepto de competencias. Memoria de DEA en ciencias de la educación, Facultad de Educación, universidad católica de Lovaina, Bélgica (2005).

Prados, J., Peterson, G. y Lattuca, L. Quality Assurance of Engineering education through Accreditation: The Impact of Engineering criteria 2000 and Its Global Influence. Journal of Engineering Education, January, 165-184 (2005).

Pinilla, Á. Aportes al concepto de competencias de América latina. Tuning América Latina. http://www.cumex.org.mx/archivos/ACERVO/Tuning.pdf (2005).Acceso: 30 de marzo (2011)

Reason, R., Terenzini, P. y Domingo, R. FIRST THINGS FIRST: Developing Academic Competence in the first year of college. Research in higher education, 47 (2), 149-175 (2006)

Roegiers, X., Pedagogía de la integración. Competencias e integración de los conocimientos en la enseñanza. Costa Rica: Coordinación Educativa y Cultural Centroamericana (2007)

http://www.ugr.es/ recfpro/rev123REC.pdf, Acceso: 11 de abril (2010)

Shuman, L., Besterfield-Sacre, M. y Mcgourty, J. The Abet "Professional Skills" - can They Be Taught? Can They Be assessed?. Journal of Engineering Education, January, 41-55 (2005)

Tuning América Latina. Reflexiones y perspectivas de la educación superior en América Latina. Informe final. Universidad de Deusto, España (2007).

http://tuning.unideusto.org/tuningal/index.php?option=com_docman\&ltemid=191\&task=view_categ ory\&catid=22\&order=dmdate_published\&ascdesc=DESC, Acceso: 31 de marzo (2010)

Vargas, F. De las virtudes laborales a las competencias clave: un nuevo concepto para antiguas demandas. Boletín Técnico Interamericano de Formación Profesional, 149, 9-24. (2000).http://www.oitcinterfor.org/public/spanish/region/ampro/cinterfor/publ/boletin/149/pdf/f_varg. pdf, Acceso: 30 de marzo (2011) 